\title{
Overdiagnosis and the cancer label
}

\author{
Richard Hurley features and debates editor
}

The BMJ

Despite Shakespeare's protestations that roses are still roses whatever you call them, nomenclature does matter. The authors of this week's Analysis make a case for stopping using the term "cancer" to describe some low risk lesions because it might be doing more harm than good (doi:10.1136/bmj.k3322).

Brooke Nickel at the University of Sydney and colleagues posit "microtumour," "abnormal cells," or "indolent lesions of low malignant potential" as alternative descriptors. Some slow growing or non-growing cancers would never cause harm if undetected. An example is low risk papillary thyroid cancer: patients receiving active surveillance do as well as those who have surgery. Long term outcomes with active surveillance of low risk ductal carcinoma in situ and localised prostate cancer are also good.

Using loaded labels such as "cancer" can make patients more worried, the authors say, which can cause them to choose more aggressive management options-with more risk of harm.

"For decades cancer has been associated with death," the authors explain. "This association has been ingrained in society with public health messaging that cancer screening saves lives. This promotion has been used with the best of intentions, but in part deployed to induce feelings of fear and vulnerability in the population and then offer hope through screening."

A change in terminology could also "help shift clinicians' perspectives and enable them to feel more comfortable recommending less invasive options to patients"-but any change would need to be assessed for benefit and harm, they say.

The names used to describe findings are but one of many potential drivers of overdiagnosis (defined as the diagnosis of a condition that will never cause symptoms or ever lead to clinical harm), as Minal S Kale and Deborah Korenstein explain in their State of the Art Review (doi:10.1136/bmj.k2820). In cases of overdiagnosis, management cannot improve health but exposes patients to risk of harm, including overtreatment and anxiety. The phenomenon is inherent to modern healthcare, which aims to diagnose and treat potentially harmful disease before it becomes clinically evident.

In the context of primary care, Kale and Korenstein detail the roots of overdiagnosis along with suggestions for managing the problem. Doctors have an ethical duty to act to minimise harm to patients, they say, but lack of awareness among doctors and patients is a fundamental obstacle to reducing overdiagnosis. "Clinicians must inform patients about overdiagnosis and incorporate it into clinical decision making."

They conclude, "There are challenges to discussing overdiagnosis with patients; the concept may be difficult to understand and some may not recognise overdiagnosis as a real problem." 